\title{
FORMULACIÓN Y VALIDACIÓN DE LA PULPA DE CAFÉ ENSILADA CON LACTOFERMENTOS, PARA LA PREPARACIÓN DE COMPLEMENTO ALIMENTICIO PARA PORCINOS.
}

\section{DEVELOPMENT AND VALIDATION COFFEE PULP ENSILED WITH LACTO-FERMENTS, FOR PREPARING FOOD SUPPLEMENT FOR SWINE}

\author{
Alba V. Díaz Corrales \\ Naxon Atahualpa Rodríguez Vallet; \\ Freidy Martín Salinas López ${ }^{\ddagger}$
}

RESUMEN: En la investigación "Formulación y validación de la pulpa de café ensilada con lacto fermentos para la preparación de un complemento alimenticio para porcinos", se planteó evaluar la pulpa de café ensilada como un alimento no tradicional en el proceso de alimentación de porcinos, valorando el nivel de consumo y aceptabilidad del producto combinado con concentrado comercial. La fase experimental se realizó en la granja ubicada en la comunidad el Naranjo del Departamento de Estelí, con cuatro cerdos de raza criolla "casco de mula" y sexo hembras en la etapa de engorde. Se pudo determinar que se puede utilizar pulpa de café ensilada como complemento a la alimentación de cerdos, cuya digestibilidad se evidencio en un valor mayor al $70 \%$. De igual manera se pudo corroborar que no puede suplir las demandas nutricionales que el concentrado comercial aporta en la etapa de engorde, por lo que se recomienda como complemento alimenticio hasta en un 15 $\%$ con concentrado comercial para cerdos. El costo de producción de 1 quintal de pulpa de café ensilada es de 272.7 córdobas netos aproximadamente, siendo este menor a un quintal de concentrado que tiene un costo de $\mathrm{C} \$ 600.00$ córdobas, teniendo los productores de café una alternativa para procesar la pulpa de café mediante el ensilaje y brindarla como complemento alimenticio para aquellas fincas que tengan crianza de cerdos o las personas que desean intentar alimentar a cerdos con complementos alimenticios más baratos que el concentrado comercial.

Palabras claves: ensilaje de pulpa de café; porcinos; suplemento alimenticio; pulpa de café; digestibilidad aparente.

ABSTRACT: In the research "Development and validation of pulp ensiled coffee lacto ferments as a dietary supplement for swine," was proposed to evaluate coffee pulp silage as a non-traditional food in the process of feeding swine, assessing the level of consumption and acceptability of the product combined with commercial food for animals. The pilot phase was conducted at the farm located in the community El Naranjo in the department of Esteli with four female swine of the race "casco de mula" in the fattening phase. It was determined that you can use coffee pulp silage to supplement pig food, the digestibility was evident to a

\footnotetext{
* Coordinadora Investigación UNI-RUACS, Nicaragua. alba.diaz@norte.uni.edu.ni - autor para la correspondencia

+ Graduado Ingeniería Agroindustrial UNI RUACS, Nicaragua

‡ Graduado Ingeniería Agroindustrial UNI RUACS, Nicaragua
} 
value greater than $70 \%$. Similarly it could corroborate that it can not fulfill the nutritional demands that the commercial food for animals brings in the fattening stage, so it is recommended as a dietary supplement by up to $15 \%$ with commercial food for swine. The production cost of one quintal of pulp ensiled coffee is 272.7 net córdobas being this less than one quintal of food for animals, which costs C\$ 600.00 córdobas, giving coffee producers an alternative to process coffee pulp by silage and provide it as a dietary supplement for those who have farms raising swine or people who want to try to feed swine with nutrional supplement cheaper than the commercial food.

Keywords: coffee pulp silage, swine, nutritional supplement, coffee pulp, apparent digestibility.

\section{INTRODUCCIÓN}

En los países productores de café, los residuos y subproductos del beneficio del café constituyen una fuente de grave contaminación y por ende, son causantes de problemas al medio ambiente, por este motivo desde mediados del siglo pasado se ha tratado de innovar métodos para utilizarlos como materia prima para la producción de abonos orgánicos, alimentos para animales, biogás, enzimas pépticas, entre otros. (Rathinavelu \& Graziosi, 2005).

Durante el proceso de beneficio húmedo de café se genera la pulpa, que al ser ensilada preserva sus características nutrimentales, tomando relevancia en el proceso de alimentación de animales. En países tropicales alcanza un valor significativo en el manejo intensivo de ganado bovino, el uso de esta pulpa de café puede alcanzar entre 20 y $30 \%$ en las raciones (Vitto, Ciria, Bonilla, \& Acenjo, 1987).

Existen en la actualidad, alternativas que permiten controlar el efecto tóxico de la pulpa que proviene del beneficio del grano de café cuando es vertido al medio ambiente, un proceso que ofrece perspectivas de poder ser aplicado es el ensilaje. Este método es una tecnología simple para manipular y dar un tratamiento a la misma la cual se produce en los beneficios húmedos en Nicaragua. Estas técnicas permiten preservar y almacenar la pulpa, manteniendo su valor nutritivo mientras se opta por un procesamiento (Noriega, Silva, \& García , 2008)

A partir de los fundamentos mencionados anteriormente se planteó el presente estudio "Validación de la pulpa de café ensilada con lacto fermentos, para la preparación de un complemento alimenticio para porcinos" Este estudio se ejecutó con la finalidad de contribuir a generar tecnologías que puedan ser utilizadas por los productores de café y de granjas de cerdos y con su implementación la reducción de los efectos ambientales que produce este desecho sólido.

\section{METODOLOGÍA}

La investigación realizada es experimental, se emplearon cuatro tratamientos los que contenían diferentes proporciones de pulpa de café ensilada y concentrado comercial, estos fueron planificados utilizando la metodología de cuadrado latino determinándose las variables: consumo de materia seca, digestibilidad, ganancia de peso. 
En la tabla 1 se presenta el diseño de cuadro latino utilizado, con el mínimo de unidades experimentales de 4 porcinos, debido a que no se contaba con recursos económicos para una mayor compra.

Tabla 1: Diseño de cuadro latino de la fase experimental

\begin{tabular}{|ccccc|}
\hline Cerdo/Tiempo & 1 & 2 & 3 & 4 \\
\hline C1 & $5 \%$ & $10 \%$ & $15 \%$ & $0 \%$ \\
\hline C2 & $10 \%$ & $15 \%$ & $0 \%$ & $5 \%$ \\
\hline C3 & $15 \%$ & $0 \%$ & $5 \%$ & $10 \%$ \\
\hline C4 & $0 \%$ & $5 \%$ & $10 \%$ & $15 \%$ \\
\hline
\end{tabular}

Las formulaciones empleadas en los cuatro tratamientos se diseñaron tomando como referencia el estudio realizado en porcinos por (Bautista, 1999), a continuación se detallan los tratamientos utilizados:

Tratamiento Testigo $1(\mathrm{~T} 1)=$ concentrado comercial $+0 \%$ de pulpa de café Tratamiento Testigo $2(\mathrm{~T} 2)=$ concentrado comercial $+5 \%$ de pulpa de café Tratamiento Testigo 3 (T3) = concentrado comercial $+10 \%$ de pulpa de café Tratamiento Testigo 4 (T4) = concentrado comercial $+15 \%$ de pulpa de café

Cada tratamiento tuvo una duración de un mes, de tal forma que todos los cerdos recibieran los 4 tratamientos, el grupo de investigadores se encargó de recolectar los datos diariamente para su posterior análisis estadístico de variancia con un modelo trifactorial sin interacciones y separación de promedios de los tratamientos de alimentación por prueba de Duncan.

\section{PRESENTACIÓN Y DISCUSIÓN DE RESULTADOS}

Aquí se presentan los resultados que se obtuvieron en la etapa experimental de la investigación y análisis respectivos.

\section{Formulación de complemento alimenticio para la alimentación de porcinos mediante el ensilaje de pulpa de café.}

Inició con el proceso de recolección de materias primas para el ensilaje, siendo el primer paso la recepción de pulpa de café fresca, para su posterior adición de melaza y lacto fermento, seguidamente compactada y almacenada en dos barriles, los cuales se sellaron herméticamente para iniciar el proceso de fermentación anaerobia durante un periodo de 100 días tiempo mínimo porque se agregó lacto fermento.

De acuerdo a (Ferrer, Páez, Chirinos, \& Zulay, 1995) en la investigación acerca del ensilaje de pulpa de café el periodo de fermentación anaeróbica puede variar entre 100 a 150 días, lo que permite disminuir el contenido de polifenoles, proteínas, cafeínas y taninos presentes en la pulpa. 
La calidad del ensilaje está en la fermentación anaeróbica disminuyendo el pH del material ensilado a valores entre 3,5 y 4,2 para evitar el desarrollo de nuevas bacterias, previniendo, de esta forma, su descomposición adicional y asegurando su conservación durante periodos largos de tiempo. En este proceso es importante propiciar un ambiente con pH de hasta 4.2, que inhiba el crecimiento de agentes patógenos y conserve las características nutricionales del producto ensilado.

Se pudo observar que el ensilaje obtenido presentaba características físicas (olor, color, forma) similar a la que posee en su estado fresco. El valor de $\mathrm{pH}$ obtenido durante la recolección estuvo en un rango de 5 a 5.5 y después del periodo de fermentación disminuyó el $\mathrm{pH}$ del material ensilado a valores de entre 4,3 y 4,4. Según Mayorga, 2005, niveles más bajos de $\mathrm{pH}$ a 4.2 en el ensilaje estaría expuesto al crecimiento y desarrollo de microorganismo y patógenos y por tanto no se conservarían las características nutricionales del producto, por eso se tuvo mucho cuidado en el control de $\mathrm{pH}$.

Para preparar 4 barriles de ensilaje de pulpa, se utilizaron 1,440 $\mathrm{kg}$ de pulpa fresca representando una composición porcentual de $92.5 \%$ de pulpa fresca, 15 litros de melaza (5\%) y $2.5 \%$ de lacto fermento. Por falta de recursos económicos no se realizaron los análisis físico-químicos para poder conocer en detalle la composición del ensilaje al agregar el lacto fermento cuyas enzimas descomponen los azucares generando ácido láctico.

En la tabla número 1 presentada en la metodología, se presenta las formulaciones del ensilaje de pulpa complementada la alimentación con concentrado comercial Almesa, proporcionado a cada cerdo durante un periodo de cuatro meses.

\section{Valoración del nivel de consumo de materia seca y digestibilidad aparente.}

Los animales mostraron buena aceptación (consumo de materia seca) y consumo del alimento proporcionado. Después de la etapa de adaptación se determinó un consumo obteniendo un valor mayor al $90 \%$ y por lo tanto no evidenciando ningún daño en el sistema digestivo del cerdo, así como no habiendo diferencia estadística entre los tratamientos, ver porcentaje de consumo de materia seca por cada tratamiento en la figura número 1.

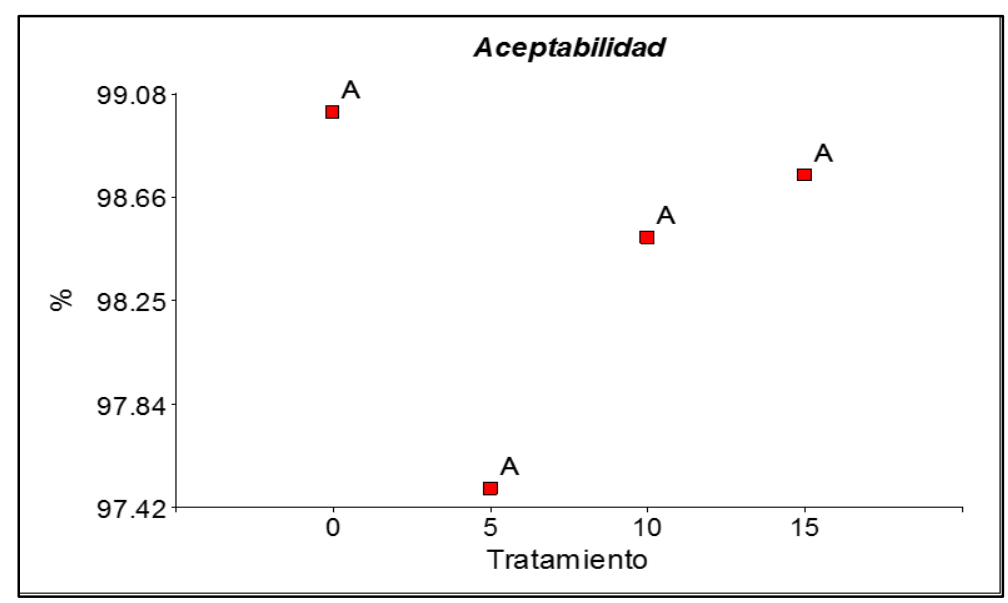

Fig. 1: Consumo de materia seca por tratamiento 
Nota: Medias con una letra común no son significativamente diferente $(p \leq 0.05)$ según prueba DGC.

Se pudo evaluar que la pulpa no genera efectos negativos (ver figura 2) en el sistema digestivo de los cerdos e incluso iniciando con el porcentaje más alto del $15 \%$ de ensilaje de pulpa de café, como fue en el caso del cerdo 3 durante el primer mes de valoración durante su alimentación.

El cálculo de la digestibilidad aparente se obtuvo aplicando la siguiente formula,

\section{DS = Consumo de materia seca - Cantidad de heces $* 100$ Consumo de materia seca}

Los resultados del análisis estadístico de varianza de cuadrado latino para los datos de heces y digestibilidad, mostraron un coeficiente de Determinación $\left(R^{2}\right)$ igual a 0.98 y 0.87 respectivamente y Coeficiente de Variabilidad (CV) igual a 7.87 y 3.06 respectivamente.

Al analizar los datos de las heces totales en cada uno de los tratamientos aplicados, se puede observar que no hay diferencia significativa entre ellos, como se puede ver en la figura número 2.

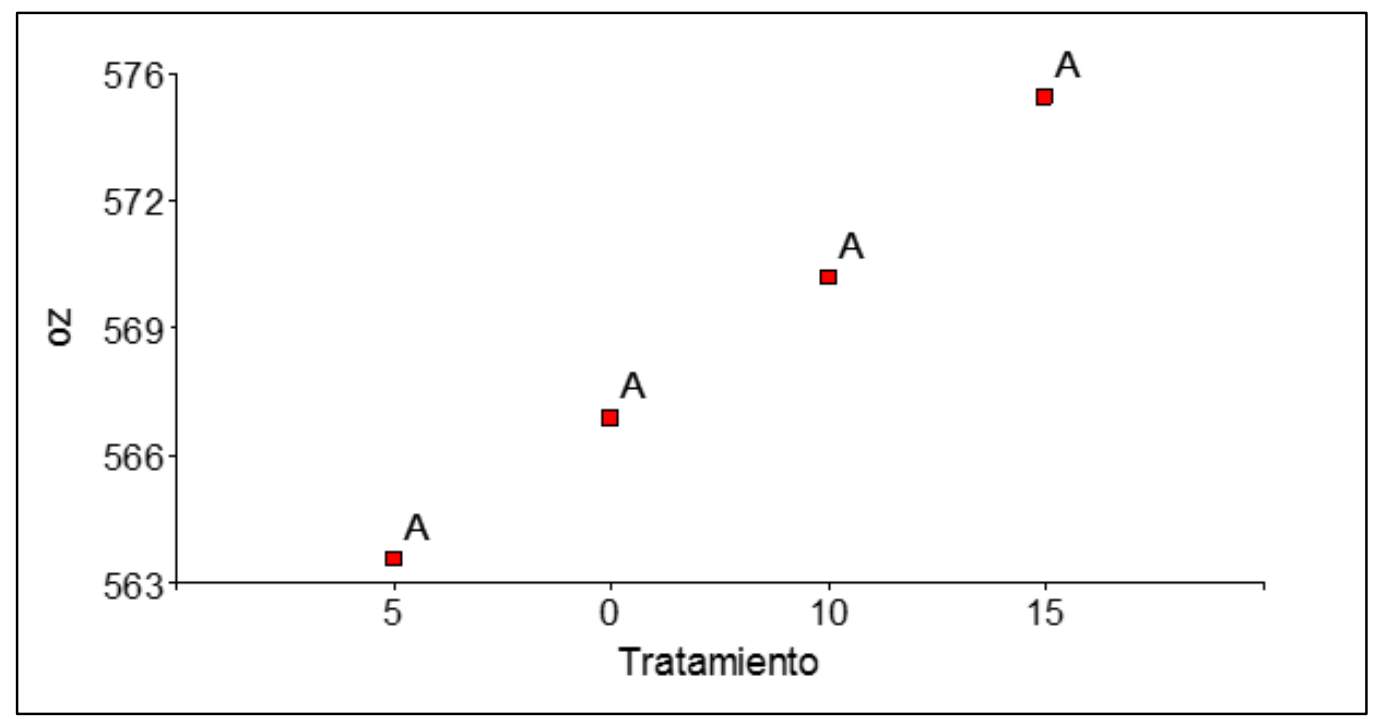

Fig. 2: Heces totales por tratamiento

Al analizar los datos de la digestibilidad aparente en cada uno de los tratamientos aplicados, se obtuvo un porcentaje mayor a $70 \%$ y no evidencia diferencia significativa entre los cuatro tratamientos, como se puede ver en la figura 3. 


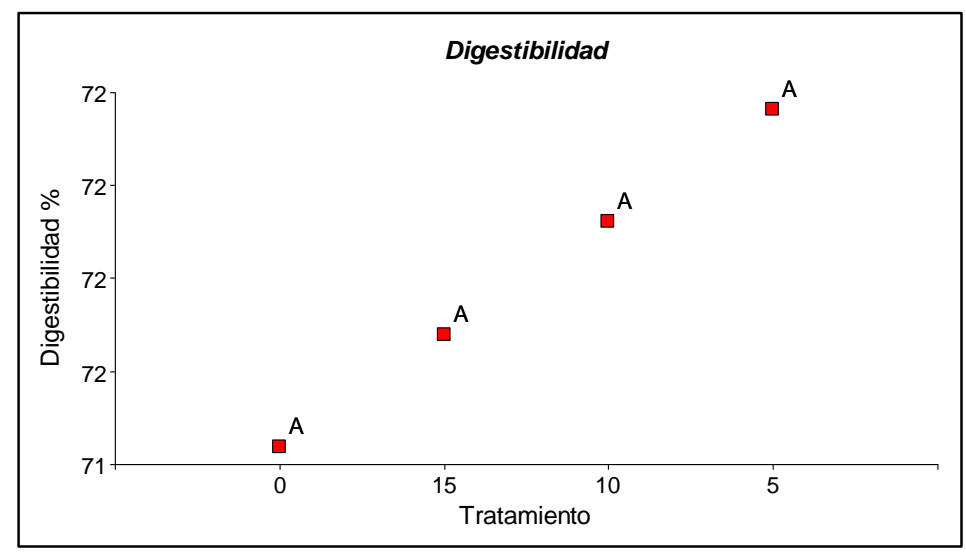

Fig. 3: Porcentaje de digestibilidad aparente por tratamiento

El valor de la digestibilidad aparente obtenido indica que el $70 \%$ del alimento proporcionado fue absorbido por los porcinos en estudio.

\section{Determinación del efecto de la pulpa de café en ganancia de peso sobre la alimentación de los cerdos.}

Tomando como referencia el diseño experimental realizado con la metodología de cuadrado latino, los mejores resultados de ganancia de peso en los porcinos en estudio se obtuvieron en el tratamiento en el que no se le proporciono ensilaje de pulpa de café, siendo este mayor hasta en un $15 \%$ mayor en peso, en el cual solamente se le proporcionó concentrado de crecimiento, como se puede ver en la figura 4.

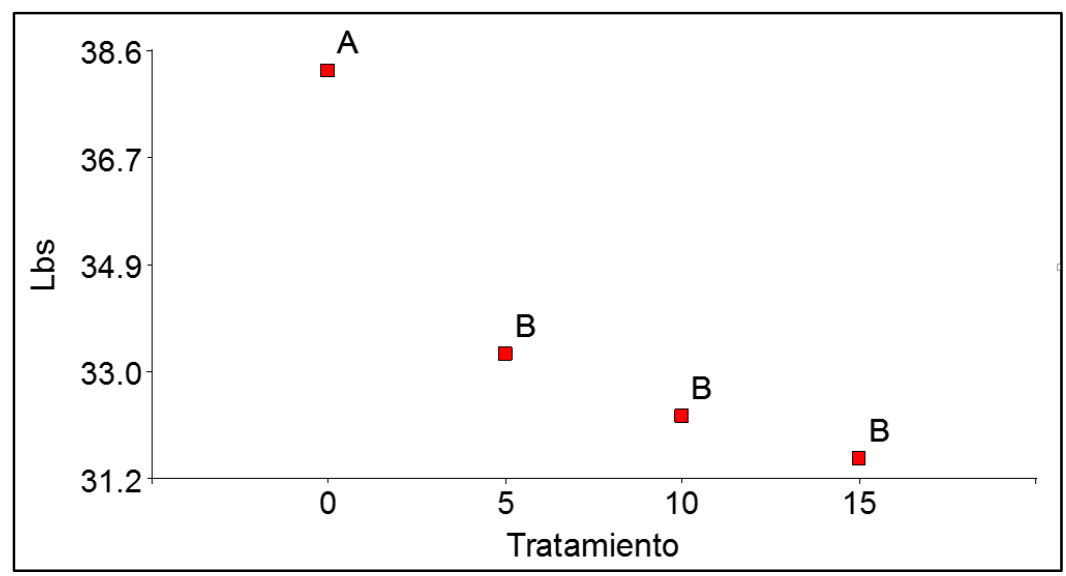

Fig. 4: Incremento de peso de acuerdo a los tratamientos empleados.

Nota: Medias con una letra común no son significativamente diferente $(p \leq 0.05)$ según prueba DGC.

Dado que el ensilaje de pulpa de café no genera efectos negativos en el sistema digestivo de los cerdos, esta es una alternativa a utilizar por parte de los productores para alimentar a los cerdos debido a que el efecto que hace el ensilaje es un crecimiento y ganancia de peso más lento en comparación a la alimentación con concentrado comercial, tomando en consideración que la curva de crecimiento de porcinos llega a un punto máximo. 
Determinar el costo de producción de la pulpa de café ensilada es un aspecto clave en el desarrollo de este estudio. El costo de producción de $1 \mathrm{Kg}$ de pulpa de café ensilada es de aproximadamente de $\mathrm{C} \$ 6.00$ córdobas netos, es decir 272.7 córdobas netas el quintal $(45.45 \mathrm{~kg})$ de ensilaje de pulpa de café.

\section{CONCLUSIONES}

Para la formulación del complemento alimenticio a partir del ensilaje de la pulpa de café se requiere como materias primas principales, pulpa de café fresca, suero láctico, melaza, yogurt natural.

Para preparar cuatro barriles de ensilaje de pulpa se utilizaron $1,440 \mathrm{~kg}$ de pulpa fresca, 15 litros de melaza, 0.5 litros de yogurt natural y 20 litros de suero láctico.

El consumo del ensilaje de pulpa en la alimentación fue mayor al $97 \%$ y con un $70 \%$ de digestibilidad. Comprobando que el ensilaje de la pulpa de café no provoca ningún efecto negativo y se puede utilizar como un complemento alimenticio para porcinos.

Al aplicar los porcentajes de 5, 10 y 15\%de ensilaje de pulpa de café en la alimentación de los porcinos, se obtuvo una disminución en el peso del 15\%, obteniendo mejores resultados al utilizar $100 \%$ concentrado de engorde.

Es posible suministrar el ensilaje de pulpa de café como un complemento alimenticio en la dieta de los cerdos no mayor a un $15 \%$ que fue lo experimentado en la dieta alimenticia durante la investigación.

El costo de producción de 1 quintal de pulpa de café ensilada es de 272.7 córdobas netos aproximadamente, siendo este menor a un quintal de concentrado que tiene un costo de C $\$ 600.00$ córdobas, teniendo los productores de café una alternativa para procesar la pulpa de café mediante el ensilaje y brindarla como complemento alimenticio para porcinos.

Con estos resultados los productores de café tienen una alternativa para procesar la pulpa de café mediante el ensilaje y brindarla como complemento alimenticio para aquellas fincas que tengan crianza de cerdos o las personas que desean intentar alimentar a cerdos con complementos alimenticios más baratos que el concentrado comercial.

\section{REFERENCIAS}

Bautista, E. B. (1999). Utilización de la pulpa de café ensilada en raciones para cerdos en crecimiento y acabado. Universidad Nacional Experimental del Táchira (UNET), Venezuela,.

Ferrer, R., Páez, G., Chirinos, M., \& Zulay, M. (1995). Ensilaje de pulpa de café. Maracaibo: Facultad de agronomia.

Mayorga, L. (2005). La pulpa de cafe: de residuo a alimento. . Recuperado el Agosto de 2012, de http://www.ugr.es/ ri/anteriores/dial03/d28-3.htm 
Noriega, A., Silva, R., \& García , M. (2008). Utilizacion de la pulpa de cafe en la alimentacion animal. Recuperado el Agosto de 2012, de http://www.scielo.org.ve/scielo.php?pid=S0798$72692008000400001 \&$ script=sci_arttex

Rathinavelu, R., \& Graziosi, G. (17 de Agosto de 2005). Posibles usos alternativos de los residuos y subproductos del cafe. Obtenido de infocafes.com/descargas/biblioteca/112.pdf

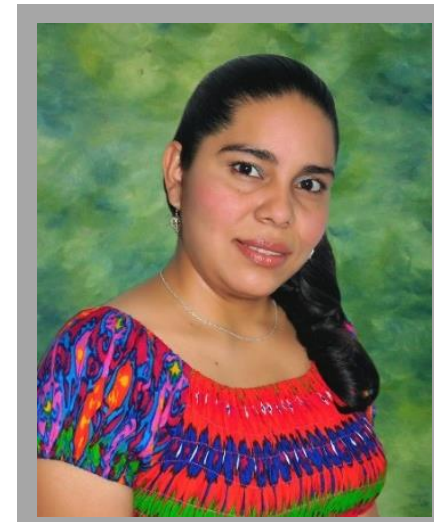

Alba Veranay Díaz Corrales: Es Ingeniero Químico, especialista en Didácticas de las Ingenierías y Arquitectura. Experiencia en Formulación y Ejecución de Proyectos de Investigación. Participación como expositora en foros y congresos a nivel nacional e internacional. Coautor de Atlas de cafés especiales de Nicaragua, Manual Técnico "Beneficio, Calidad y Denominación de Origen y publicación de artículos científicos en la revista científica de la UNI-Norte "EI Higo". Docente e investigadora en la temática Denominación de Origen. Asesora en la aplicación de herramientas TGP. Tutora de tesis monográficas. 